\title{
A Scalable Framework for Multi-Robot Tele-Impedance Control
}

\author{
Virginia Ruiz Garate ${ }^{* 1}$, Soheil Gholami*1,2, and Arash Ajoudani ${ }^{1}$
}

\begin{abstract}
In this manuscript, we present an online scalable tele-impedance framework, which enables the individual and collaborative control of multiple different robotic platforms. The framework provides an intuitive low-cost interface with visual feedback and a SpaceMouse, through which the operator can define the desired task-level trajectories and impedance profiles. With a simple SpaceMouse click, the user can switch between the robots and the collaborative operation mode. The control, subsequently, manages the distribution of the required parameters into the involved robots. Thanks to the introduced virtual hand concept where each robot is defined as a finger, new robots can be easily added or removed via their kinodynamic parameters. The proposed framework was evaluated with three different experiments: a simulated auscultation on a mock-up patient, a cooperative task where a robot drives the patient on a wheelchair and a different robot performs the auscultation, and a collaborative task where two robots relocate a container. The results demonstrate the capabilities of the framework in terms of adaptability to different robotic platforms, the number of robots involved, and the task requirements. Additionally, quantitative and subjective analysis of 12 subjects showed how the developed interface, even in the presence of inaccurate visual feedback, allowed a smooth and accurate execution of the tasks.
\end{abstract}

Index Terms-Tele-impedance, multi-robot control, medical application.

\section{INTRODUCTION}

$\mathbf{R}$ OBOTIC teleoperation is a widely studied domain with multiple application potential. Clinical care (e.g., telemedicine and decontamination [1], [2]), rehabilitation (e.g. bilateral-arm exoskeleton [3]) disaster response (e.g., postearthquake intervention [4]), and reconnaissance (e.g., supervising compliance with voluntary quarantines and monitoring inaccessible environments [5]), are among the applications where telerobots can make a real difference. However, despite its historical research background, robotic teleoperation has not made its way into resolving our complex societal problems. An example is the recent Covid-19 pandemic, where telerobots could have provided unique opportunities for limiting the spread and the impact of such a highly-contagious disease across all continents. Instead, medical staff and relevant authorities were at the front-line of this crisis, performing patients' reception, diagnosis, etc.

In response to this shortfall and to bring telerobotic solutions to real environments, efforts must be devoted to the develop-

This work was supported by the European Research Council (ERC) starting grant Ergo-Lean, Grant Agreement No. 850932

* : Both authors contributed equally to this work

$1 \mathrm{HRI}^{2}$ Lab, Istituto Italiano di Tecnologia, Genoa, Italy, Emails: Virginia.Ruiz@iit.it, Soheil.Gholami@it..it

${ }^{2}$ NearLab, Dept. of Electronics, Information and Bioengineering, Politecnico di Milano, Milan, Italy

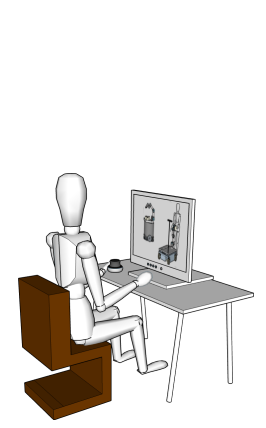

(a)

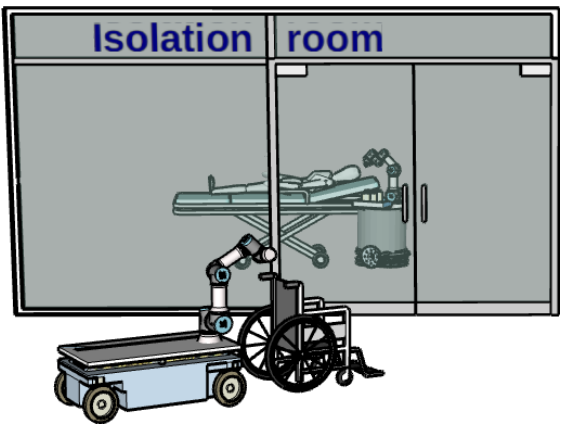

(b)
Figure 1: Conceptual illustration of multi-robot teleoperation in a medical application

ment of reliable, adaptive, scalable, and intuitive interfaces that are operable and acceptable by non-experts. In fact, until now, most of the research studies done in this domain have focused on issues related to the stability and robustness of the teleoperation systems [6], to the control of remote interaction [7], [8], and to the feedback modalities (e.g., haptic signals [9] or virtual reality [10]). Yet, few studies focused on the usability aspects of the teleoperation frameworks [11], while no attention has been paid to their scalability to various challenging interaction scenarios (see Fig. 1 that shows an example of telerobots being used in a medical context).

Indeed, the possibility to have multiple robots controlled by one operator seems to be an additional crucial requirement, especially in medical or industrial contexts, where different robots must take diverse roles to perform different actions. This reconfigurability of the robotic modules can contribute to reducing equipment idle times and the associated costs. Nonetheless, it poses the problem of scalability of the control systems, calling for an adaptable software framework able to cope with changing platforms and tasks, number of robots, and collaborative scenarios.

As a similar but not identical problem, previous works [12], [13], studied the formulation of object-level bi-manual manipulation. While the former work allows to distribute the outputs to the end-effector as desired, in the latter, torque outputs directly manipulate joints' positions and stiffness without having direct control on the end-effector states. Similarly in [14], the authors provide an object impedance control for cooperative multiple-arm manipulator systems based on Nakamura's multifingered hand controller [15], providing end-point force-plusacceleration commands. These works however focus on traditional dual-arm systems, which are coupled together to a torso or fixed on a base, and therefore cannot move and interact independently with the environment. In [16], independent 
movements of both robots are studied, while the arms were still attached to a fixed surface. Furthermore, experiments were performed only with simulated planar manipulators rigidly holding an object. Works dealing with independent systems that function together to modify an object trajectory and/or impedance are mostly related to human-robot cooperation [17], [18], [19]. The example presented in [20] allows the independent control of one or two robot arms fixed on a table to grasp and manipulate an object. Yet, single and dual-arm strategies are independent in this case and the Cartesian stiffness for the end-effectors of the robots is defined separately for each of them, resulting in an inefficient scalable approach.

Another fundamental aspect is the effectiveness and easiness-of-use of the teleoperation interfaces, which are crucial to assure their easy adoption and usage by nonexperts. Several works have tackled this issue, both from feedback/supervision and user input points of view. For instance, recently, virtual reality [21], [22], augmented reality [23], [24], and mixed reality [25] have found their way to the teleoperation systems by providing a better perception of the remote environment. However, in such scenarios, the users must be equipped with special sensors and devices, which can be tiresome and frustrating during prolonged operations. Also from the input viewpoint, complex and expensive systems have been developed to map the user's motions to the telerobot's ones, such as optical motion capture systems [26], inertial motion capture systems [27], [28], and skeleton's motion extraction by using cameras [29]. Still, there exist some interfaces to accomplish teleoperation tasks in a cheaper and easier way. For instance, in [30] authors evaluate the use of two different SpaceMouse (3D mouse) devices versus the traditional keyboard and mouse. This study shows how, in particular for people with motor disabilities, the participants find it more challenging to perform the tasks with the keyboard and mouse than with the 3D devices, and easier with the SpaceMouse ${ }^{\circledR}$ Compact rather than SpaceMouse ${ }^{\circledR}$ Pro version.

Towards a unified solution to the above wide-spread problems, in this manuscript we propose a novel teleoperation paradigm for the remote impedance control of multi-robots in different operational configurations. The framework provides an intuitive interface with visual feedback and a 3D mouse, enabling an operator to switch in real-time between different control modes (from a single robot arm to collaborative robots) and motion modes (translation or rotation), depending on the object to handle and the task to execute. Additionally, it provides the users with the ability to adjust the Cartesian stiffness values in arbitrary axes, based on a desired physical interaction (e.g., compliant when dealing with a patient or stiff when moving a wheelchair). This concept, known as teleimpedance control [7], is integrated in the proposed interface to improve robot adaptation to remote physical interaction requirements while eliminating the need for the troublesome force-feedback loops [31]. The interface requires minimal training time ( 5 to 10 minutes, across 12 subjects) highlighting its easiness-of-use.

The controller itself presents a novel approach, which addresses the multi-robot grasping problem from a system- atic and software-reconfigurable point of view, extending the multi-finger grasping formulation to a multi-robot interaction framework. This allows us to use the powerful control theories of robotic hand manipulation [32], [33], [34] to solve the challenge of multi-robot control and co-manipulation. In our scheme, a virtual hand model is created where each of the robotic modules is represented by a virtual finger with several degrees of freedom (DoF) and kinematic specifications (with or without base mobility). The manipulation is defined at the object-level, by specifying the trajectories and the impedance profiles needed to perform a task. These specifications are mapped to the necessary profiles for the virtual fingertips by means of the grasp matrix [34]. To do so, the virtual hand is continuously updated, transforming the independent movements of the modules into coupled motions of the equivalent virtual fingers and vice-versa. The foremost contribution of this approach lies on the possibility to use a unified control framework to command different types of robots either individually or in combination.

The potential of the proposed framework is evaluated through three different sets of experiments. First, the basic capabilities of the proposed controller and the intuitiveness of the interface are assessed through the use of a fixed-base manipulator for a simulated auscultation of a patient via the Fixed Manipulation (FM) control mode. The second set of experiments shows the potential of the framework to smoothly switch between the control of different platforms (control modes) regulating their locations and Cartesian stiffness in real-time. The executed task consists of driving a wheelchair with a mock-up patient using of a mobile manipulator (using the Mobile Manipulation (MM) control mode), towards a fixed-base manipulator to cooperatively execute an auscultation task. The third experiment demonstrates the Collaborative Manipulation $(C M)$ capabilities of the control framework together with the switching between the different control modes. The executed task involves the grasping of a box with potential bio-hazardous products and its placement in a new location.

\section{Methodology}

An schematic representation of the proposed framework is illustrated in Fig. 2. The main building blocks of this structure are the scalable multi-robot impedance control and the user interface (UI). The former is composed of the multi-robot control and the robotic modules blocks. The UI allows the operator to monitor the remote environment through the visual feedback provided by the installed RGB cameras. Moreover, the interaction forces/torques are shown during the execution of the task allowing the user to modify the motions (with the 3D mouse) and stiffness values (with the Graphical User Interface (GUI)). The actual equilibrium poses for the robots' low-level controllers are generated by the constructed virtual hand in the multi-robot control block. The following subsections explain in detail these main building blocks.

\section{A. User interface (UI)}

The operator is provided with a UI allowing him/her to control and monitor the system with a 3D mouse and a GUI. 


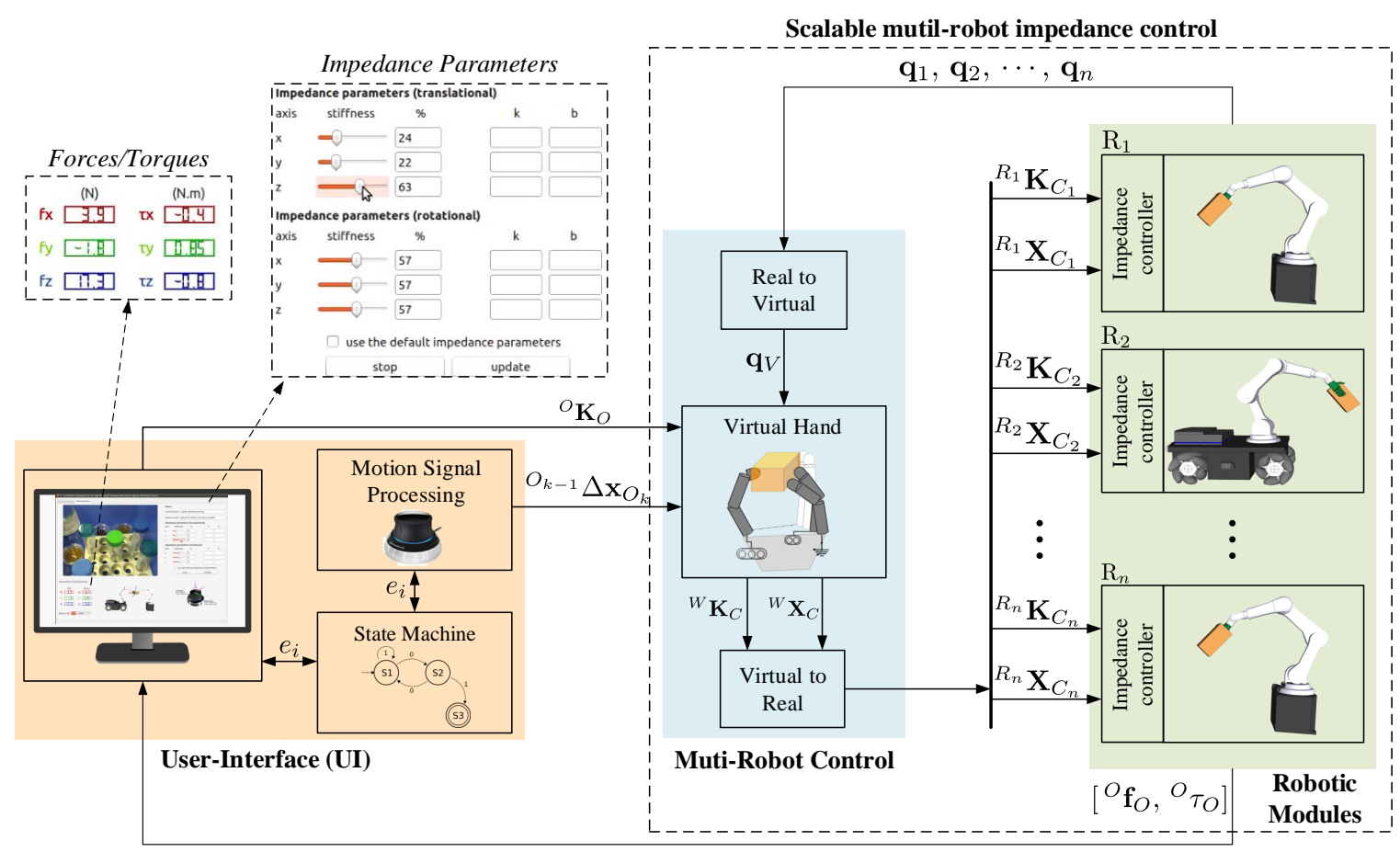

Figure 2: Main building blocks of the scalable framework for multi-robot tele-impedance control. This is developed by means of a ROS robotic middleware and the Qt framework. The "Interaction Forces/Torques" and "Impedance Parameters Settings" widgets of the GUI are highlighted for clarity. The remote operator utilizes the "User Interface (UI)" to command motions and impedance settings to the object. These are transformed into the required parameters for each of the robots employed throughout the task by means of the "Multi-Robot Control", which is based on the concept of the virtual hand.

The employed mouse (3Dconnexion [35]) has two buttons and a 6 DoF motion sensor. Left and right buttons are set to drive a state machine (Fig. 2) enabling the user to switch between different control modes (i.e., FM, MM, and CM) and motion modes (i.e., translation and rotation), to activate the setting of the Cartesian impedance parameters, and to close/open the gripper/hand of each robot. The $6 \mathrm{DoF}$ motion axis of the $3 \mathrm{D}$ mouse is used by the operator to generate the reference trajectories for robots' low-level controllers. These DoFs are highly coupled and very sensitive. Therefore, a motion signal processing algorithm is designed to generate a smooth and decoupled reference trajectory for the telerobots.

First, the mouse's raw motions, updated based on the exerted force/torques of the user, are encapsulated in a vector as $\Delta \mathbf{x}=[\Delta \mathbf{p}, \Delta \boldsymbol{\epsilon}]^{T} . \Delta \mathbf{p} \in \mathbb{R}^{3}$ and $\Delta \boldsymbol{\epsilon} \in \mathbb{R}^{3}$ represent the translational and rotational displacements, respectively. $\Delta \mathrm{x}$ is then normalized to a generic signed percentage vector $\Delta \mathbf{x}^{\dagger}$, with all its six elements in the range of $[-100,100] \%$. Afterwards, the motion mode is checked by the algorithm, setting $\Delta \epsilon$ or $\Delta \mathrm{p}$ to zero when being on the translation or rotation motion mode, respectively. Next, a moving average filter is applied with a window $\chi \in \mathbb{R}^{6 \times N}$ that is populated based on the current $\Delta \mathbf{x}_{k}^{\dagger}$ and its last $N-1$ values. Thus, at time instant $k$ we have $\chi_{k}=\left[\begin{array}{llll}\Delta \mathbf{x}_{k-N-1}^{\dagger}, & \cdots, & \Delta \mathbf{x}_{k-1}^{\dagger}, & \Delta \mathbf{x}_{k}^{\dagger}\end{array}\right]$. The average value of each motion axis $j$ of the mouse over the last $N$ samples is then calculated:

$$
\Delta \overline{\mathbf{x}}_{j_{k}}=\frac{1}{N} \sum_{i=k-N-1}^{k} \chi_{j, i}, \quad j \in\{1, \cdots, 6\} .
$$

$N$ is chosen both to account for the filtering requirements and the bandwidth of the low-level controllers. The maximum value of $\Delta \overline{\mathrm{x}}$ together with its corresponding movement axis $j^{\star}$ are retrieved while the other output values are set to 0 . The desired displacement vector of the object is then generated $\Delta \mathbf{x}_{d}=\left[\Delta \mathbf{p}_{d}, \Delta \boldsymbol{\epsilon}_{d}\right]^{T}$, where only the $j^{\star}$-th element is nonzero. $\Delta \mathbf{x}_{d}$ is finally converted to a desired motion in the object frame ${ }^{O_{k-1}} \Delta \mathbf{x}_{O_{k}}=\left[{ }^{O_{k-1}} \Delta \mathbf{p}_{O_{k}},{ }^{O_{k-1}} \Delta \boldsymbol{\epsilon}_{O_{k}}\right]^{T}$, based on the pre-set maximum values $\Delta \mathbf{p}_{M}$ and $\Delta \epsilon_{M}$ for translational and rotational displacements, respectively. These are chosen taking into account the sensors' resolution and manipulability requirements.

Regarding the feedback to the user, a Qt-based [36] GUI (Fig. 2), allows the operator to observe the remote environment and accomplish the desired tasks. The provided GUI also informs the user of the current status of the system (control mode, motion mode, battery level of the robots, etc.) and interaction forces/torques. Additionally, the GUI enables the operator to set the desired stiffness parameters after activating the configuration mode with the 3D mouse buttons. More specifically, the user can modify the diagonal elements of the Cartesian object-level stiffness ${ }^{O} \mathbf{K}_{O} \in \mathbb{R}^{6 \times 6}$ in terms of percentage of a minimum and maximum values. After the values are updated, the system resumes the operation in its last control mode, retrieved from the state machine memory. It should be noted that the stiffness modification is possible during each control and motion mode at any point of the teleoperation. 



(b)

Figure 3: (a) Equivalence between the robots' frames and contact points in the real world and virtual hand system for an example case with four robotic modules. (b) Three possible control modes, from left to right: FF, MM, and CM. Frames are defined in Table III of Appendix A.

\section{B. A scalable framework for multi-robot control}

This block generates the desired object-level pose and Cartesian impedance profiles to regulate the robots' motions and interaction with the environment. To do so, it receives the object displacement ${ }^{O_{k-1}} \Delta \mathbf{x}_{O_{k}}$ and stiffness values ${ }^{O} \mathbf{K}_{O}$ as inputs from the UI and converts them into the corresponding commands for the robots' end-effectors (Fig. 2).

The controller aims to capitalize on the powerful tools of robotic hand grasping by transforming the set of involved robots into modelled fingers (virtual fingers) of a reconfigurable virtual hand (Fig. 3a). Hence, robotic modules can be easily added or removed based on the task requirements. Each robotic module, which is now represented by a virtual finger, is added to the virtual hand (Fig. 3b) by means of the corresponding Denavit-Hartenberg (DH) parameters. To fully define this virtual hand, the location of the fingers' base must be determined. While the locations of the fixed-base robot modules are known, for the mobile modules the fingers' base are placed using virtual joints ( 2 prismatic joints for the linear translations plus 1 revolute joint for the rotation). A conceptual model of the system including $n$ different robotic modules for the different control modes is illustrated in Fig. $3 \mathrm{~b}$ (for CM control mode, an example case is shown).

Thanks to the definition of this virtual hand, known grasping paradigms [32], [33], [34] can be now applied to translate the object-level displacements and stiffness profiles coming from the UI block into the corresponding values for the fingertips of the virtual fingers. These values are then mapped to the necessary commands for the robotic modules (Fig. 2), where the desired trajectories and stiffness profiles of the embedded low-level Cartesian impedance controllers are updated.

The main sub-elements of the multi-robot control block are discussed in detail in the following subsections:

1) Object-level motion: the operator is able to command the desired movement of the object with respect to (w.r.t.) its own frame (at previous time instant $k-1$ ) by means of the motion signal processing output ${ }^{O_{k-1}} \Delta \mathbf{x}_{O_{k}}$. This desired motion is then expressed in the virtual hand system, where the new pose of the object frame $\left\{O_{k}\right\}$ w.r.t the virtual reference (world) frame $\{W\}$ is obtained by the following homogeneous transformation matrix ${ }^{W} \mathbf{T}_{O_{k}}$ :

$$
{ }^{W} \mathbf{T}_{O_{k}}={ }^{W} \mathbf{T}_{O_{k-1}}{ }^{O_{k-1}} \mathbf{T}_{O_{k}}
$$

$O_{k-1} \mathbf{T}_{O_{k}}$ is updated based on the displacement output of the UI block ${ }^{O_{k-1}} \Delta \mathbf{x}_{O_{k}}=\left[{ }^{O_{k-1}} \Delta \mathbf{p}_{O_{k}}{ }^{O_{k-1}} \Delta \boldsymbol{\epsilon}_{O_{k}}\right]^{T}$ :

$$
\begin{aligned}
{ }^{{ }_{k-1}} \mathbf{T}_{O_{k}} & =\left[\begin{array}{c|c}
{ }^{O_{k-1}} \mathbf{R}_{O_{k}} & { }^{O_{k-1}} \Delta \mathbf{p}_{O_{k}}^{T} \\
\hline \mathbf{0}_{1 \times 3} & 1
\end{array}\right] \\
{ }^{O_{k-1}} \mathbf{R}_{O_{k}} & =R_{x}\left(\Delta \boldsymbol{\epsilon}_{1}\right) R_{y}\left(\Delta \boldsymbol{\epsilon}_{2}\right) R_{z}\left(\Delta \boldsymbol{\epsilon}_{3}\right) .
\end{aligned}
$$

$\Delta \epsilon_{1}, \Delta \epsilon_{2}$, and $\Delta \epsilon_{3}$ are the elements of ${ }^{O_{k-1}} \Delta \boldsymbol{\epsilon}_{O_{k}}$. Note that $O_{k-1} \mathbf{R}_{O_{k}}=\mathbf{I}_{3 \times 3}$ and $O_{k-1} \Delta \mathbf{p}_{O_{k}}^{T}=\mathbf{0}_{3 \times 1}$ in the translation and rotation motion modes, respectively. This new object pose ${ }^{W} \mathbf{T}_{O_{k}}$ is then mapped to the desired pose of each fingertip $\left\{C_{i}\right\}$ w.r.t. $\{W\}$ :

$$
{ }^{W} \mathbf{T}_{C_{i k}}={ }^{W} \mathbf{T}_{O_{k}}{ }^{O_{k}} \mathbf{T}_{C_{i_{k}}} .
$$

${ }^{O} \mathbf{T}_{C_{i k}}$ represents the transformation matrix from the $i$ th contact point $\left\{C_{i}\right\}$ to the object frame $\left\{O_{k}\right\}$. In this work, fixed grasping contacts are considered, thus, ${ }^{O}{ }_{k} \mathbf{T}_{C_{i k}}=$ ${ }^{O_{0}} \mathbf{T}_{C_{i 0}}$, being able to retrieve it at the beginning of each control mode's activation by using the same equation (4). ${ }^{W} \mathbf{T}_{C_{i k}}$ is obtained at any instant from direct kinematics using the virtual hand system's DH parameters and the actual joint positions of the robots. It should be noted that ${ }^{O} \mathbf{T}_{C_{i 0}}=\mathbf{I}_{4 \times 4}$ in case of single-robot scenario, as we assume the object is rigidly grasped by the end-effector. When multiple robots are collaborating, several approaches can be used to determine the pose of the object in the virtual world ${ }^{W} \mathbf{T}_{O_{k}}$. Our method tries to simplify the amount of needed sensory systems by following a similar approach to [37]. This considers the object initially located in the center of the fingertips when the grasp happens ${ }^{W} \mathbf{p}_{O_{0}}=\frac{1}{n} \sum_{i=1}^{n}{ }^{W} \mathbf{p}_{C_{i 0}}$, where $n$ is the number of the robotic modules. Also, ${ }^{W} \mathbf{R}_{O_{0}}$ is defined by creating a plane crossing the fingertip locations. This strongly depends on the number of the hand's virtual fingers. A particular case with two robotic modules is presented in Section III.

2) Object-level impedance: the impedance characteristics of the grasped object need to be tuned to obtain the desired interaction with the environment and thus execute the required tasks successfully. To do so, we focus on updating the desired stiffness values through the GUI by specifying the translational and rotational diagonal stiffness values expressed in $\{O\}$ $\left({ }^{O} \mathbf{K}_{T} \in \mathbb{R}^{3 \times 3}\right.$ and ${ }^{O} \mathbf{K}_{R} \in \mathbb{R}^{3 \times 3}$, respectively). These matrices are then combined into the single block-diagonal objectlevel stiffness matrix ${ }^{O} \mathbf{K}_{O} \in \mathbb{R}^{6 \times 6}$. It should be noted that the damping values of the impedance model are updated according to the processed user-defined stiffness values such that the passivity conditions of the Cartesian impedance controllers are satisfied [38]. Also, the inertia-shaping technique is not employed in the impedance controllers' implementation (see Section II-C). 
To be able to transform the desired stiffness into commands for the individual robots, first ${ }^{O} \mathbf{K}_{O} \in \mathbb{R}^{6 \times 6}$ should be converted to $\{W\}[34]$ :

$$
\begin{aligned}
{ }^{W} \mathbf{K}_{O} & ={ }^{O} \mathbf{Z}_{W}^{T}{ }^{O} \mathbf{K}_{O}{ }^{O} \mathbf{Z}_{W}, \\
{ }^{W} \mathbf{Z}_{O} & =\left[\begin{array}{cc}
{ }^{W} \mathbf{R}_{O} & \mathbf{0}_{3 \times 3} \\
\mathbf{0}_{3 \times 3} & { }^{W} \mathbf{R}_{O}
\end{array}\right] .
\end{aligned}
$$

${ }^{W} \mathbf{R}_{O}$ is the rotation matrix from $\{O\}$ to $\{W\}$ which can be extracted from ${ }^{W} \mathbf{T}_{O_{k}}$ (2). The desired object stiffness needs then to be translated to the corresponding $n$ virtual fingertips' stiffness matrices. From the robotic grasp theory, the stiffness on an object ${ }^{W} \mathbf{K}_{O}$ can be defined as [34]:

$$
{ }^{W} \mathbf{K}_{O}=\mathbf{G}{ }^{W} \mathbf{K}_{\mathbf{C}} \mathbf{G}^{T} .
$$

$\mathbf{G} \in \mathbb{R}^{6 \times n}$ is the grasp matrix transforming the stiffness from the $n$ fingertips into the object by means of the skewsymmetric matrices of the virtual fingertip positions w.r.t. the object's center $\left(\mathbf{S}_{C_{i}} \in \mathbb{R}^{3 \times 3}\right.$ for the $i$-th contact) [34]:

$$
\mathbf{G}=\left[\begin{array}{cc|cc|c|cc}
\mathbf{I}_{3 \times 3} & \mathbf{0}_{3 \times 3} & \mathbf{I}_{3 \times 3} & \mathbf{0}_{3 \times 3} & \cdots & \mathbf{I}_{3 \times 3} & \mathbf{0}_{3 \times 3} \\
\mathbf{S}_{C_{1}} & \mathbf{I}_{3 \times 3} & \mathbf{S}_{C_{2}} & \mathbf{I}_{3 \times 3} & \cdots & \mathbf{S}_{C_{n}} & \mathbf{I}_{3 \times 3}
\end{array}\right] .
$$

For the sake of clarity, matrices referring to the $\{W\}$ will carry no upper-script in what follows, e.g. $\mathbf{K}_{\mathrm{C}} \stackrel{\text { def }}{=}{ }^{W} \mathbf{K}_{\mathrm{C}}$. $\mathbf{K}_{\mathrm{C}} \in \mathbb{R}^{6 n \times 6 n}$ is the block diagonal matrix containing the Cartesian stiffness values at the $n$ contact points:

$$
\mathbf{K}_{C}=\left[\begin{array}{cccc}
\mathbf{K}_{C_{1}} & \mathbf{0}_{6 \times 6} & \cdots & \mathbf{0}_{6 \times 6} \\
\mathbf{0}_{6 \times 6} & \mathbf{K}_{C_{2}} & \cdots & \mathbf{0}_{6 \times 6} \\
\vdots & \vdots & \ddots & \vdots \\
\mathbf{0}_{6 \times 6} & \mathbf{0}_{6 \times 6} & \cdots & \mathbf{K}_{C_{n}}
\end{array}\right] .
$$

$\mathbf{K}_{\mathrm{C}_{i}}$ is defined as:

$$
\mathbf{K}_{\mathrm{C}_{i}}=\left[\begin{array}{ll}
\mathbf{K}_{C_{i}, t} & \mathbf{K}_{C_{i}, c} \\
\mathbf{K}_{C_{i}, c}^{T} & \mathbf{K}_{C_{i}, r}
\end{array}\right], \quad i \in[1, n] .
$$

$\mathbf{K}_{C_{i}, t} \in \mathbb{R}^{3 \times 3}, \mathbf{K}_{C_{i}, r} \in \mathbb{R}^{3 \times 3}$, and $\mathbf{K}_{C_{i}, c} \in \mathbb{R}^{3 \times 3}$ represent the translational, rotational, and coupled stiffness sub-matrices of the $i$-th contact point.

Writing (6) analytically, and using (7)-(9), the following set of equations are obtained:

$$
\begin{gathered}
\mathbf{K}_{O, t}=\sum_{i=1}^{n} \mathbf{K}_{C_{i}, t}, \mathbf{K}_{O, c}=\sum_{i=1}^{n}\left(\mathbf{K}_{C_{i}, c}+\mathbf{K}_{C_{i}, t}^{T} \mathbf{S}_{C_{i}}^{T}\right), \\
\mathbf{K}_{\mathrm{O}, \mathrm{r}}=\sum_{i=1}^{n}\left(\mathbf{K}_{C_{i}, r}+\mathbf{S}_{C_{i}} \mathbf{K}_{C_{i}, t}^{T} \mathbf{S}_{C_{i}}^{T}+\mathbf{S}_{C_{i}}^{T} \mathbf{K}_{C_{i}, c}\right. \\
\left.+\mathbf{K}_{C_{i}, c}^{T} \mathbf{S}_{C_{i}}^{T}\right) .
\end{gathered}
$$

$\mathbf{K}_{O, t}, \mathbf{K}_{O, r}$, and $\mathbf{K}_{O, c} \in \mathbb{R}^{3 \times 3}$ are the symmetric matrices of the translational, rotational, and coupled terms of the desired object stiffness.

In the general case, (10) presents $6 \times n$ equations and $n \times 3 \times 6$ unknowns, being the system under-determined. optimization methods depending on the individual robots (e.g., limited stiffness) can be used to solve this system of equations. Instead, in the case of single robot (FM and MM control modes), the system becomes fully-determined $(n=1)$ and can be solved analytically. For the following, when dealing with several robotic units, we will consider an homogeneous distribution of the forces, assigning symmetrically the stiffness parameters. In this case, the equations in (10) are straightforward to solve. Still, the limit values of the Cartesian stiffness for each of the robots must be taken into account, setting a minimum that allows to overcome the internal friction of the robot and a maximum that does not generate large torques. These constraints can be expressed as:

$$
\begin{aligned}
& n \mathbf{K}_{C_{i}, t_{\text {min }}}<\mathbf{K}_{O, t}<n \mathbf{K}_{C_{i}, t_{\max }}, \\
& n\left(\mathbf{K}_{C_{i}, r_{\text {min }}}+\mathbf{K}^{\ddagger}\right)<\mathbf{K}_{O, r}<n\left(\mathbf{K}_{C_{i}, t_{\max }}+\mathbf{K}^{\ddagger}\right), \\
& \mathbf{K}^{\ddagger}=\sum_{i=1}^{n}\left(\mathbf{S}_{C_{i}} \mathbf{K}_{C_{i}, t}^{T} \mathbf{S}_{C_{i}}^{T}+\mathbf{S}_{C_{i}}^{T} \mathbf{K}_{C_{i}, c}+\mathbf{K}_{C_{i}, c}^{T} \mathbf{S}_{C_{i}}^{T}\right) .
\end{aligned}
$$

$\mathbf{K}_{C_{i}, t_{\min }}, \mathbf{K}_{C_{i}, t_{\max }}, \mathbf{K}_{C_{i}, r_{\min }}$ and $\mathbf{K}_{C_{i}, r_{\max }}$ represent the allowed minimum and maximum translational and rotational Cartesian stiffness of the robot, respectively. If the resulting stiffness for the end-effectors reaches these limits, it is saturated. This affects the resulting object-level stiffness that is recomputed (10) and displayed in the UI (Fig. 2). Therefore, to avoid undesired behaviours, the object-level stiffness must be carefully chosen. For the translational stiffness $\mathbf{K}_{O, t}$, it is straightforward to set a value that respects the robot Cartesian stiffness limits (11). However, for the rotational stiffness, the definition of a particular object translation stiffness $\mathbf{K}_{O, t}$ and the position of the grasping points change the boundaries through $\mathbf{K}^{\ddagger}$ (11). Knowing the task preliminary, simulations can be performed checking approximate grasping points and using $\mathbf{K}_{O, t_{\text {min }}}$ and $\mathbf{K}_{O, t_{\max }}$ (or better the actual used $\mathbf{K}_{O, t}$ if known beforehand), to obtain general boundaries for these values. To ease this procedure for the user, we set the object stiffness as a percentage between the resulting minimum and maximum values assuring that the constraints are always satisfied.

3) Conversion between the virtual and real system: once the desired motion displacements of the virtual fingertips ${ }^{W} \mathbf{X}_{C}$ (extracted from ${ }^{W} \mathbf{T}_{C_{i k}}$ ) and their stiffness matrix ${ }^{W} \mathbf{K}_{C}$ are generated, they need to be mapped into the corresponding commands for the robotic modules (Fig. 2, virtual to real conversion block).

For the end-effector pose, a transformation from the virtual reference frame $\{W\}$ to the base frame of each of the robotic modules $\left\{R_{i}\right\}$ should be applied:

$$
{ }^{R_{i}} \mathbf{T}_{C_{i}}={ }^{R_{i}} \mathbf{T}_{W}{ }^{W} \mathbf{T}_{C_{i}} .
$$

${ }^{R_{i}} \mathbf{T}_{W}$ is the transformation matrix between the virtual hand's world frame $\{W\}$ and the $i$-th robot base frame $\left\{R_{i}\right\}$. For the fixed-base robot units, this matrix is constant and can be retrieved from the initial configuration. For the locomanipulation units, however, $\left\{R_{i}\right\}$ is usually set as the initial location when the robot is switched on. Therefore, to retrieve ${ }^{R_{i}} \mathbf{T}_{W}$, the following sequence of conversions are applied:

$$
{ }^{R_{i}} \mathbf{T}_{W}={ }^{R_{i}} \mathbf{T}_{P_{i}}{ }^{P_{i}} \mathbf{T}_{M_{i}}{ }^{M_{i}} \mathbf{T}_{C_{i}}{ }^{W} \mathbf{T}_{C_{i}}^{-1} .
$$

As the mobile manipulators are made of two robotic modules, the mobile platform and the manipulator itself, ${ }^{R_{i}} \mathbf{T}_{P_{i}}$ represents the transformation between the current $i$-th mobile 
platform's location $\left\{P_{i}\right\}$ and its original reference frame $\left\{R_{i}\right\}$, which can be constructed from the robot's odometry data. Then, ${ }^{P_{i}} \mathbf{T}_{M_{i}}$ is the constant transformation matrix between the location of the manipulator's reference frame $\left\{M_{i}\right\}$ and the mobile platform's frame $\left\{P_{i}\right\} .{ }^{M_{i}} \mathbf{T}_{C_{i}}$ is the transformation between the virtual fingertip's location $\left\{C_{i}\right\}$ and $\left\{M_{i}\right\}$ (same as the virtual finger), which can be obtained straightforward from the direct kinematics using the finger's DH parameters. $R_{i} \mathbf{T}_{W}$ is a constant matrix so it can be obtained at the beginning of the operation by using (13).

Regarding the Cartesian stiffness, the one computed for the virtual fingertips from (10) should be transformed to the reference frame of each robot $\left\{R_{i}\right\}$. Similarly to (5): ${ }^{R} \mathbf{K}_{C_{i}}={ }^{W} \mathbf{Z}_{R_{i}}^{T} \mathbf{K}_{C_{i}}{ }^{W} \mathbf{Z}_{R_{i}}$, with ${ }^{W} \mathbf{Z}_{R_{i}}$ being the blockdiagonal matrix composed of ${ }^{W} \mathbf{R}_{R_{i}}$ which is extracted from (13).

\section{Robotic modules}

Once the new end-effector poses and Cartesian stiffness are obtained, desired torques $\left(\boldsymbol{\tau}_{q}\right)$ for the fixed-base and mobile manipulators are computed through each robot's Cartesian impedance controllers. Our method focus on obtaining these reference poses and Cartesian stiffness, thus exploiting the already implemented impedance controllers. These can vary from one manipulator to another. Generally speaking, for fixed-base manipulators, a Cartesian impedance controller such as [39] can be used, while for the case of mobile manipulators, a whole-body impedance controller such as [27], [40] should be employed. A brief introduction on the employed impedance controllers in our experiments are given in the Appendix C.

When the robots' control systems are updated, the virtual hand needs to be adjusted based on the robotic modules' sensory data (Fig. 2, real to virtual conversion block). These are transformed from the real robotic joint positions $\left[\mathbf{q}_{1}, \mathbf{q}_{2}, \cdots, \mathbf{q}_{n}\right]$ into a set of joint positions for the virtual hand system $\left(\mathbf{q}_{V}\right)$. For the fixed-base manipulation, the read angles are directly mapped to the DH parameters of the virtual hand. Instead, for the mobile-base manipulators, like previously pointed in Section II-B, the readings of the mobile platform are converted into a set of sequential virtual joints accounting for the rotation and translation of the mobile platform. These joints' states can be extracted from the transformation matrix locating the base of the robotic manipulator w.r.t the virtual world: ${ }^{W} \mathbf{T}_{M_{i}}={ }^{W_{i}} \mathbf{T}_{R_{i}}{ }^{R_{i}} \mathbf{T}_{P_{i}}{ }^{P_{i}} \mathbf{T}_{M_{i}}$.

\section{EXPERIMENTS}

The effectiveness of the proposed control framework was evaluated through three sets of experiments pivoting around medical applications. In these experiments, the Franka Emika Panda robotic manipulator [41] and the MObile Collaborative robotic Assistant (MOCA [27]) were employed as the fixedbase and mobile-base manipulators, respectively. MOCA was equipped with the Pisa/IIT SoftHand [42] during all the experiments. For Franka, a mock-up auscultation ${ }^{1}$ tool was used as its end-effector throughout the first two experiments. For the last experiment (collaborative scenario), however, its default gripper was used. The heterogeneity of the platforms and grippers aims to evidence the potential of the framework to work with diverse hardware platforms. Next subsections describe in detail the three experimental set-ups.

\section{A. Experimental set-up description}

The objective of the first experimental set-up is to evaluate the easiness-of-use and intuitiveness of the developed teleoperation user-interface (3D mouse and the GUI) in a simulated medical context. To do so, 12 healthy subjects were asked to carry out a simulated auscultation operation with a fixed-base robotic arm using the FM control mode. A human manikin was located on a table as a mock-up test patient, where two different points were highlighted as target auscultation points for consistency: one in the upper part of the chest and other in the lower one (Fig. 4). The subjects were asked to remotely move the robot from the start pose, contact the target points (always in the same order), and maintain the contact for 5 s (simulating auscultation time), while watching the visual feedback and interaction force/torque through the GUI. The operators carried out the task in two different conditions: (i) with maximum fixed impedance parameters $(100 \%$, see Section III-B), and (ii) with self-selected impedance parameters, i.e., the user was able to freely update the stiffness values during the task. Subjects' ages were in the range of 21 to 38 years old, being 8 men and 4 women. They were also asked to define themselves as "expert" or "naive" users in the tele-impedance control: 7 operators chose expert whereas 5 described themselves as "naive" users. Before starting the real task, each subject was trained for about 5 minutes to use the interface and get habituated to the robot's motions. They were also informed about basic notions of compliance and stiffness and guidelines were given about how to set the impedance parameters for the execution of the task. In general, they were asked to set a higher compliance when they wanted to avoid high-force impacts, and to be stiffer if they preferred very accurate movements. For this experiment, task execution time is evaluated together with the maximum and average impact forces exerted on the patient. To assess the workload imposed on the users, we also asked the participants to compile a NASA Task Load Index (NASA-TLX) [43] at the end of the experiment. This was accompanied by two more questions: (i) with which stiffness setting you found the task easier to accomplish? and (ii) with which stiffness setting you felt the patient was safer?

The second experimental set-up aims to show the potential of the framework for cooperative tasks by smoothly switching between the FF and MM control modes to make both robots work towards the completion of a common task. As it is

\footnotetext{
${ }^{1}$ The selection of the auscultation task in our experimental set-up was based on the challenging interaction requirements between the robot and the future patients, and on the feedback we received from the medical experts in the treatment of Covid-19 patients. In fact, this type of examination in Covid-19 isolation areas has been considered very risky for the medical staff, since it may expose human respiratory system to the virus through ears.
} 

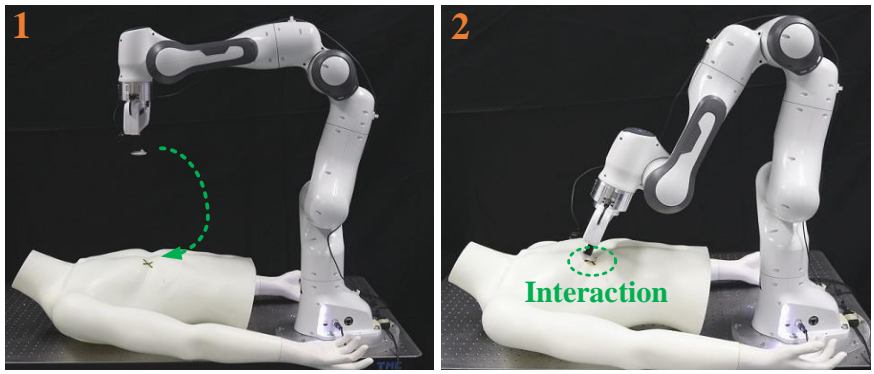

Figure 4: First experiment. 1) displays the robot in the initial configuration and one of the auscultation targets. 2) the execution of the simulated auscultation task and the end-effector's interaction with the manikin's body.
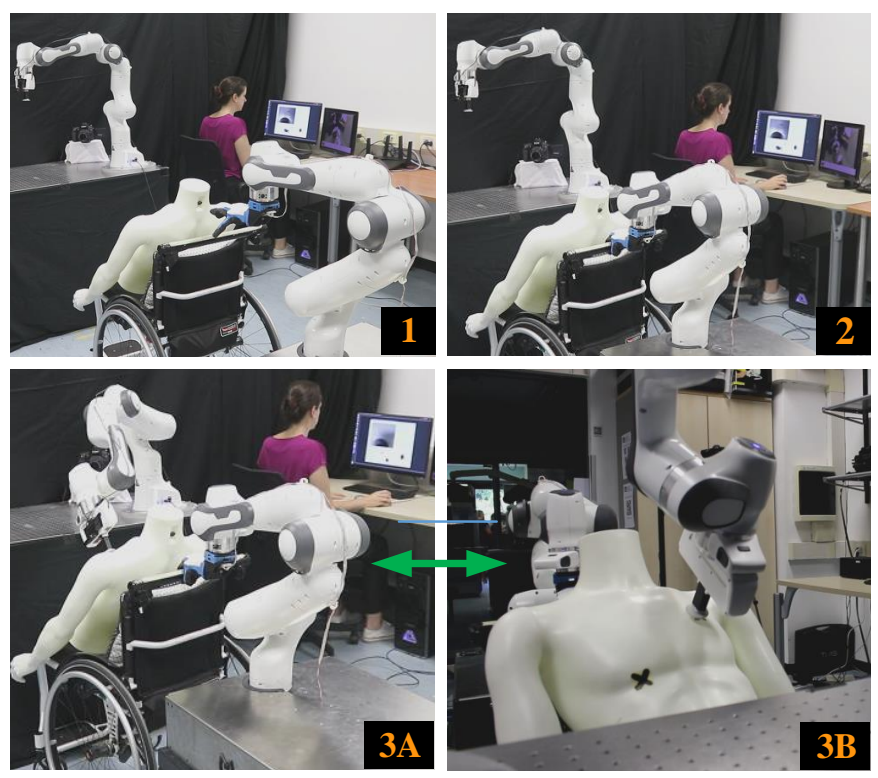

Figure 5: Second experiment. Three significant moments of the experiment process are shown: 1) the initial configurations of the robots, 2) MOCA's pose after the user operates it to grasp the handle of the wheelchair and push it towards the desk, and 3) the simulated auscultation task. (Pictures 3A and 3B show two different viewpoints of this task)

shown in Fig. 5, the human manikin was put on a wheelchair to simulate another possible chest auscultation scenario. One "expert" operator executed this experiment, where a mobile manipulator had to be first driven towards the wheelchair, located in a different position ahead of the robot. The robot grasped the wheelchair handle and pushed it towards the fixedbase robot by means of the MM control mode based on the operator's commands. When the mobile manipulator reached the desired place near the table, the user switched to the FM control mode of the framework by simply pressing the mouse's button and carried out the auscultation task for one of the check points. It should be noted that the impedance parameters were updated by the user at different instants of the task execution depending on the requirements of the actions to be performed. For this experiment we evaluate the influence of the stiffness settings on the interaction forces/torques and the smooth switching between the different control modes.

The third experimental set-up investigated the collaborative capabilities of the proposed framework (CM). A remote pickand-place task was executed by employing fixed and mobile manipulators sequentially and simultaneously to grasp and transport a box. The box was filled with medical tools simulating bio-hazardous materials and located on a table near the fixed-base robotic arm (Fig. 6-1). In this task, an "expert" operator first moved the fixed-base manipulator to one of the box handles and grasped it by means of the FM control mode (Fig. 6-2). Next, the operator switched to the MM control mode to drive the mobile manipulator towards the box and grasp the other box's handle (Fig. 6-3). Then, the CM was activated by the user, moving the box towards a desired location in a nearby trolley (Fig. 6-4 to Fig. 6-7). After placing the box, the operator opened the grippers. Then she tele-operated the fixedbase manipulator to a safe place using the FM control mode (Fig. 6-8). Finally, the operator switched again the control mode to MM to use the mobile-base manipulator for pulling the trolley backwards towards a new location (Fig. 6-9 to Fig. 6-10). During all the operations, the user constantly checked the interaction force/torque values through the GUI, deciding to open/close the grippers depending on the interaction status. In this experiment we evaluate the capability of the system to smoothly switch between the different control modes and to effectively distribute the desired collaborative object-level motions and impedance parameters to the individual robots.

\section{B. Experimental parameters}

The virtual hand model described in Section II-B is created based on the SynGrasp toolbox [44] for MATLAB, which we partly translated into a $\mathrm{C}++$ library available at [45]. The $\mathrm{DH}$ parameters of the virtual hand are given in Appendix B.

Having two virtual fingers, the object initial location in (4) is estimated with ${ }^{W} \mathbf{p}_{O_{0}}$ and the rotation matrix ${ }^{W} \mathbf{R}_{O_{0}}=$ $\left[\mathbf{r}_{x}, \mathbf{r}_{y}, \mathbf{r}_{z}\right] \in S O(3)$ from the location of the end-effectors of both robotic systems ${ }^{W} \mathbf{p}_{C_{1}}=\left[x_{1}, y_{1}, z_{1}\right]$ and ${ }^{W} \mathbf{p}_{C_{2}}=$ $\left[x_{2}, y_{2}, z_{2}\right]$ at the instant of switching to the CM control mode (Section II-B1). $\mathbf{r}_{x}$ is placed by joining both end-effectors pointing from the first to the second robot. The other two axes are defined to create the orthogonal frame. As we only have two points, we define $\mathbf{r}_{y}$ such that the resulting $\mathbf{r}_{z}$ will be pointing upwards in the global frame. Therefore, the object frame is fully defined from:

$$
\begin{aligned}
{ }^{W} \mathbf{p}_{O_{0}} & =\frac{1}{2}\left({ }^{W} \mathbf{p}_{C_{1}}+{ }^{W} \mathbf{p}_{C_{2}}\right), \\
\mathbf{r}_{x} & =\frac{{ }^{W} \mathbf{p}_{C_{2}}-{ }^{W} \mathbf{p}_{C_{1}}}{\left\|{ }^{W} \mathbf{p}_{C_{1}}+{ }^{W} \mathbf{p}_{C_{2}}\right\|}, \\
\mathbf{r}_{y} & =\mathbf{r}_{x} \times[0,0,-1], \quad \mathbf{r}_{z}=\mathbf{r}_{x} \times \mathbf{r}_{y} .
\end{aligned}
$$

To completely define the equations in Section II, in (1), $N$ was set to 15 samples as a trade-off between delay and filtering requirements; and $\Delta \mathbf{p}_{M}=[0.003,0.003,0.003]^{T} \mathrm{~m}$ for translational and $\Delta \epsilon_{M}=[0.3,0.3,0.3]^{T}$ rad for rotational motion modes. These maximum values rendered the whole movement relatively slow, which we chose as a compromise between the needed time to execute the task and the safety for the inexperienced users. This speed can be easily increased as users become more comfortable with the system.

For (10) and (11), $\mathbf{K}_{O, t_{\max }}$ diagonal values were set to 1000 $\mathrm{N} / \mathrm{m}$, and for $\mathbf{K}_{O, r_{\max }}$ to $75 \mathrm{Nm} / \mathrm{rad} . \mathbf{K}_{O, t_{\text {min }}}$ diagonal values were set to $50 \mathrm{~N} / \mathrm{m}$, and for $\mathbf{K}_{O, r_{\text {min }}}$ to $1 \mathrm{Nm} / \mathrm{rad}$. These were 

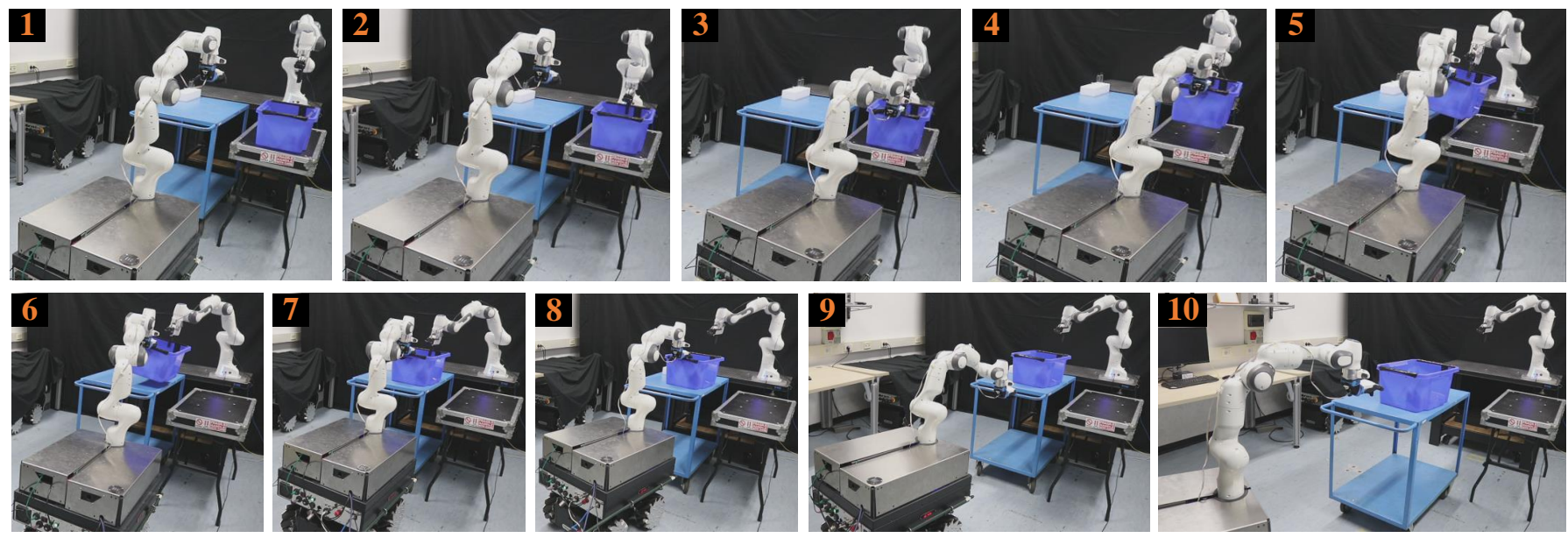

Figure 6: Third experiment. Snapshots taken from a video during the execution of the task. Pictures 1-2 show the first use of the FM control mode to grasp the box from one of its handles, after which the MM control mode is activated to grasp the other handle of the box (picture 3). The operator then switched to CM control mode and placed the box in the new location (pictures 4-7). Then, the user switched back to FM control mode to release the box (picture 8), and finally used the MM control mode to open the hand and grasp the trolley's handle in order to drive it towards a new location (pictures 9-10).

chosen as the minimum common values allowing the robots to overcome the friction effects when being teleoperated with the previously defined settings of the $3 \mathrm{D}$ mouse.

\section{Experimental Results}

Fig. 4 shows the initial position and one of the simulated auscultation poses for the first experiment. Table I displays the experimental results for the 12 subjects in terms of the task execution time, selected stiffness, and resulting interaction forces. The time needed to execute the task both with $100 \%$ of the stiffness values and with self-selected ones are displayed. As expected, varying the stiffness during the task execution made the whole process slower, being the median $\Delta t=47.95$ $\%$ faster with fixed stiffness values. Still, the slower subject with fixed values $\left(S_{4}\right)$ took more time than the fastest one $\left(S_{5}\right)$ even when the user self-selected the parameters. Regarding the interaction forces during the simulated auscultation task, the average values were retrieved for both contact points, together with the maximum peak forces for the full period of the task. As the forces are obtained in the end-effector frame, we focus on the force applied along the auscultation tool, thus, only the vertical force $f_{z}$ is shown. Applied contact forces during the auscultation period were more than $50 \%$ lower when subjects self-regulated the stiffness, for which they generally set the vertical stiffness values to lower than $30 \%$. One anomaly is $S_{5}$, who kept the stiffness to $100 \%$ also for the self-regulated case. Despite the high-variability in the selected stiffness and applied forces, most subjects applied less force against the chest of the patient when they reduced the vertical stiffness. As exceptions, $S_{7}$ exerted more force for the first auscultation point with the self-selected stiffness, even though it was set to only $10 \%$ of the maximum, and also $S_{9}$ and $S_{10}$ experienced a similar situation for the second auscultation point. Still, for these cases, the maximum recorded peak force was larger with bigger stiffness values. Just $S_{6}$ had a higher impact force at an instant with the lower stiffness value, though the operator later stabilized to lower contact forces during the auscultation period.

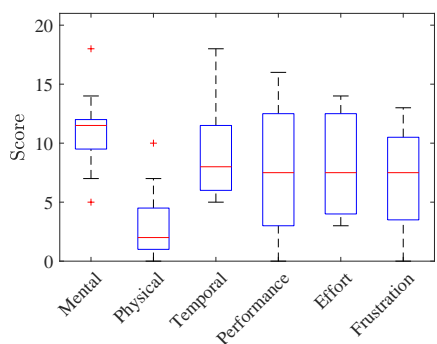

(a)

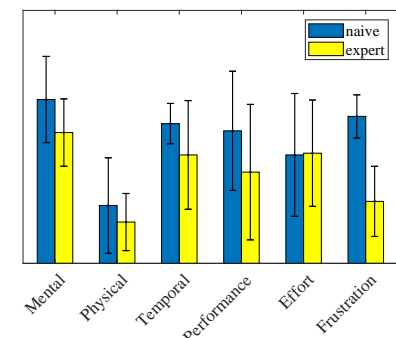

(b)
Figure 7: (a) General box-plot of the NASA-TLX subjective test with 12 participants. The red "+" symbol indicates the outliers. (b) NASA-TLX subjective results divided in "naive" and "expert" users.

Fig. 7 shows the NASA-TLX results regarding the userinterface usability. As could be expected, subjects ranked the task as low physically demanding, being the higher score in the mental demand. The temporal, performance, effort, and frustration scores show a generally low-demanding task which still can be improved. Indeed, most users had several suggestions to refine the interface, being the most repeated one the inconvenience of having to trigger the stiffness configuration from the mouse to be able to then set it in the GUI. Also, several "naive" subjects reported it difficult to choose the stiffness values, as they were not familiar with the concept. One of them suggested to present the users with several preset options, e.g.: low, medium, high to make the choice easier for the operator. Indeed, from Fig. 7b, it can be seen how experienced users reported lower demand for all the questions. Just the score of effort index was very similar between lowexperienced and "expert" users.

Regarding the two additional questions, 6 of the 12 subjects reported that it was easier to perform the task with the high pre-set stiffness values, among which were 3 of the "naive" subjects, and the other half found it easier with the selfselected values. Nonetheless, 8 of the 12 subjects felt that the subject was safer when they were able to decrease the stiffness values before contact. Only one "expert" user reported feeling safer with higher stiffness values.

For the second experiment Fig. 5 shows three representative 
Table I: Execution times and interaction forces during the experiments. $T_{1}, T_{2}$ : time to execute the task with fixed and self-selected stiffness values, respectively. It took $\Delta t(\%)$ less time to execute the task with fixed stiffness than with self-selected one. $f_{z_{1}}$ and $f_{z_{1}}$ (max): peak vertical force exerted with fixed and self-selected stiffness, respectively. $f_{z_{11}}$ and $f_{z_{21}}(\mu \pm \sigma)$ : average vertical forces exerted on the first auscultation position with fixed and self-selected stiffness, respectively. $f_{z_{21}}$ and $f_{z_{22}}(\mu \pm \sigma)$ : average vertical forces exerted on the second auscultation position with fixed and self-selected stiffness, respectively. The median and IQR (difference between the 75th and the 25th percentiles of the data) values are also displayed. $k_{z}$ : percentage of the used stiffness in the vertical direction (along the tool) when applying the self-selected stiffness. For the other case, $k_{z}=100 \%$.

\begin{tabular}{|c|c|c|c|c|c|c|c|c|c|c|c|}
\hline Subject & $T_{1}[\mathrm{~s}]$ & $T_{2}[\mathrm{~s}]$ & $\Delta t[\%]$ & $\begin{array}{c}f_{z_{1}}[\mathrm{~N}] \\
(\max )\end{array}$ & $\begin{array}{c}f_{z_{2}}[\mathrm{~N}] \\
(\max )\end{array}$ & $\begin{array}{c}f_{z 11}[\mathrm{~N}]^{*} \\
(\mu \pm \sigma)\end{array}$ & $\begin{array}{c}f_{z 21}[\mathrm{~N}]^{*} \\
(\mu \pm \sigma)\end{array}$ & $k_{z}[\%]$ & $\begin{array}{c}f_{z 12}[\mathrm{~N}]^{*} \\
(\mu \pm \sigma)\end{array}$ & $\begin{array}{c}f_{z 22}[\mathrm{~N}]^{*} \\
(\mu \pm \sigma)\end{array}$ & $k_{z}[\%]$ \\
\hline$S_{1}$ & 83.76 & 171.84 & 51.26 & 13.24 & 7.25 & $11.10 \pm 0.064$ & $6.18 \pm 0.036$ & 33 & $13.14 \pm 0.038$ & $7.13 \pm 0.033$ & 38 \\
\hline$S_{2}$ & 115.40 & 245.92 & 53.07 & 26.15 & 6.69 & $26.03 \pm 0.038^{* * *}$ & $6.42 \pm 0.063$ & 21 & $12.39 \pm 0.033$ & $5.045 \pm 0.041^{* *}$ & 20 \\
\hline$S_{3}$ & 118.20 & 213.44 & 44.62 & 90.22 & 35.16 & $89.18 \pm 0.180$ & $10.01 \pm 0.044$ & 13 & $43.19 \pm 0.070$ & $35.055 \pm 0.062$ & 11 \\
\hline$S_{4}$ & 138.32 & 204.00 & 32.19 & 18.21 & 5.66 & $5.30 \pm 0.036$ & $1.63 \pm 0.025$ & 0 & $17.097 \pm 0.036$ & $3.75 \pm 0.042$ & 0 \\
\hline$S_{5}$ & 59.68 & 102.36 & 41.69 & 64.37 & 24.79 & $64.16 \pm 0.062$ & $23.35 \pm 0.024$ & 11 & $37.21 \pm 0.110^{* *}$ & $16.021 \pm 0.039$ & 100 \\
\hline$S_{6}$ & 64.20 & 109.63 & 41.44 & 13.17 & 39.87 & $12.97 \pm 0.070$ & $2.87 \pm 0.029$ & 0 & $11.028 \pm 0.055$ & $4.50 \pm 0.037$ & 0 \\
\hline$S_{7}$ & 99.32 & 279.08 & 64.41 & 20.74 & 17.89 & $14.20 \pm 0.037$ & $17.14 \pm 0.031$ & 10 & $20.57 \pm 0.048$ & $3.35 \pm 0.055$ & 11 \\
\hline$S_{8}$ & 78.44 & 117.80 & 33.41 & 24.85 & 5.64 & $10.75 \pm 0.058$ & $5.49 \pm 0.036$ & 32 & $7.17 \pm 0.042$ & $5.26 \pm 0.022$ & 28 \\
\hline$S_{9}$ & 123.16 & 165.99 & 25.80 & 38.51 & 20.62 & $14.91 \pm 0.067$ & $10.068 \pm 0.032$ & 20 & $10.087 \pm 0.055$ & $18.43 \pm 0.180$ & 20 \\
\hline$S_{10}$ & 87.40 & 285.16 & 69.35 & 25.11 & 22.33 & $24.79 \pm 0.045$ & $22.19 \pm 0.034$ & 47 & $18.23 \pm 0.046$ & $20.004 \pm 0.037$ & 24 \\
\hline$S_{11}$ & 99.76 & 216.56 & 53.93 & 45.51 & 39.87 & $40.28 \pm 0.570$ & $26.93 \pm 0.069$ & 28 & $45.35 \pm 0.061$ & $39.65 \pm 0.031$ & 31 \\
\hline$S_{12}$ & 47.92 & 104.00 & 53.92 & 22.85 & 7.72 & $22.66 \pm 0.085$ & $4.25 \pm 0.030$ & 21 & $14.60 \pm 0.120$ & $5.97 \pm 0.080$ & 26 \\
\hline median & 93.36 & 187.92 & 47.94 & 24.99 & 19.26 & 18.79 & 8.22 & - & 15.85 & 6.55 & - \\
\hline IQR & 5.48 & 117.52 & 16.50 & 22.53 & 23.01 & 21.12 & 14.79 & - & 17.18 & 14.44 & - \\
\hline
\end{tabular}

* To retrieve the mean and standard deviation of contact forces, the moment in which the operator starts a simulated auscultation period of $5 \mathrm{~s}$ is detected by checking the point at which he/she releases the mouse. The first second after that point is neglected to allow some stabilization and the following 3 seconds are considered for the computation.

** The computation time interval considered for these cases is different due to undesired movements performed by the operators. The following time periods are used from the beginning of the auscultation (without deleting $1 \mathrm{~s}$ in the start): for $S_{2}$ in $f_{z_{11}} 1.5$ [s], for $S_{2}$ in $f_{z_{22}} 3$ [s], and for $S_{5}$ in $f_{z_{21}} 2$ [s].

instants: 1) initial set-up, 2) mobile manipulator has driven the wheelchair towards the final location, and 3) simulated auscultation task. The last picture displays another view of the final auscultation task. Fig. 8 shows the results for this experiment in terms of end-effector displacement and Cartesian stiffness for both robots, together with the measured interaction forces. The subject first used the MM control mode to drive the MOCA robot towards the wheelchair handle with the default stiffness values. Once an increase of force was detected in the $z$ direction, indicating contact with the handle, the user closed the robotic hand to grasp the wheelchair $(t \approx 125 \mathrm{~s})$. The operator decreased the stiffness in $z$-axis to be compliant to possible irregularities on the floor and increased the stiffness in the $x$-axis and $y$-axis to allow a more accurate follow-up of the desired trajectory. Still, in terms of rotation, stiffness around the $z$-axis was set to a high value, to prevent the wheelchair from turning. It can be observed how these changes immediately influenced the interaction forces, decreasing in the vertical direction and increasing the in the horizontal plane. Also the torques around the $z$-axis significantly increased. Next, the operator drove the wheelchair towards the fixedbase robotic arm, and once arrived switched the control mode to FM. The user then regulated the end-effector location to a nearby position to the auscultation point, after which the stiffness was tuned to become compliant along the longitudinal axis of the tool and in all rotational directions $(t \approx 230 \mathrm{~s})$. A short auscultation task was performed being the contact forces around $10 \mathrm{~N}$ and the torques lower than $1 \mathrm{Nm}$.

A snapshot sequence of the third experiment is displayed in Fig. 6. In this test, we selected $30 \%$ of the Cartesian stiffness in all axes. This was applied independently to the robots when operating in the FM and MM control modes, and to the grasped object when in $\mathrm{CM}$ control mode. Stiffness values are shown in Table II. Taking into account $\mathbf{K}_{C_{i}, t}$ and $\mathbf{K}_{C_{i}, r}$ minimum and maximum values (Section III-B), $30 \%$ for $\mathrm{FM}$ and $\mathrm{MM}$ translated into $335 \mathrm{~N} / \mathrm{m}$ and 23.2
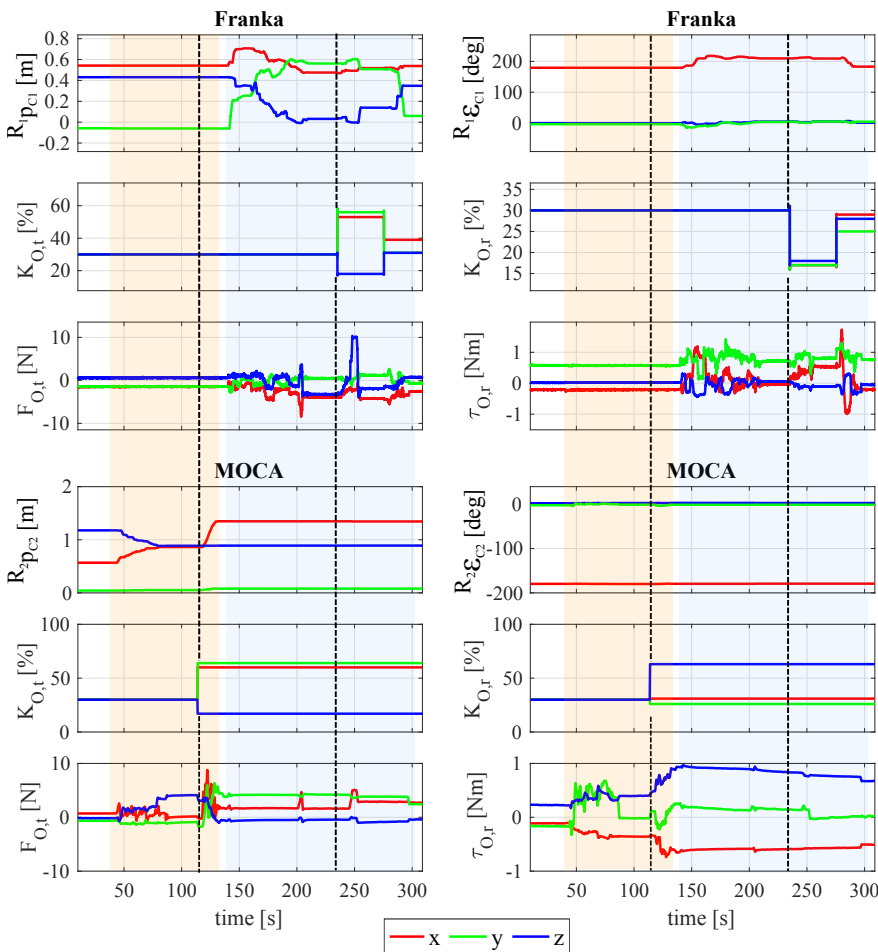

Figure 8: Second experimental results. End-effector displacement, Cartesian stiffness, and interaction forces/torques for the fix-base robot (Franka) and the mobile manipulator (MOCA). Left column: translation; right column: rotation. The vertical dotted lines are the moments when the stiffness values were modified. Red- and blue-shadowed areas represent the operator using the MM and FM control modes, respectively.

$\mathrm{Nm} / \mathrm{rad}$, respectively. When in $\mathrm{CM}$ control mode, taking into account the maximum and minimum Cartesian stiffness values, according to (10) and (11) the following minimum and maximum object-level stiffness values in translation are retrieved: $\mathbf{K}_{O, t_{\max }}=2000 \mathrm{~N} / \mathrm{m}$ and $\mathbf{K}_{O, t_{\min }}=100 \mathrm{~N} / \mathrm{m}$. Yet, for the rotation terms, the contact point location must be taken into account, as translation forces can create torques 
Table II: Translational and rotational stiffness values during the execution of the third experiment. Used matrices are diagonal, and thus the diagonal terms in translation $\left[x_{t}, y_{t}, z_{t}\right]$ and rotation $\left[x_{r}, y_{r}, z_{r}\right]$ are displayed.

\begin{tabular}{|c|cccc|}
\hline axis & $K_{O}$ & $K_{O}$ & $K_{F M}$ & $K_{M M}$ \\
& {$[\%]$} & {$[\mathrm{Nm}]-[\mathrm{Nm} / \mathrm{rad}]$} & {$[\mathrm{Nm}]-[\mathrm{Nm} / \mathrm{rad}]$} & {$[\mathrm{Nm}]-[\mathrm{Nm} / \mathrm{rad}]$} \\
\hline$x_{t}$ & 30 & 670 & 335 & 335 \\
$y_{t}$ & 30 & 670 & 335 & 335 \\
$z_{t}$ & 30 & 670 & 335 & 335 \\
$x_{r}$ & 30 & 46.4 & 23.2 & 23.2 \\
$y_{r}$ & 30 & 588.92 & 23.2 & 23.2 \\
$z_{r}$ & 30 & 588.92 & 23.2 & 23.2 \\
\hline
\end{tabular}

trough lever arms. Therefore: $\mathbf{K}_{O, r_{\max }}=150 \mathrm{Nm} / \mathrm{rad}$, and $\mathbf{K}_{O, r_{\text {min }}}=2 \mathrm{Nm} / \mathrm{rad}$ for the $x$-axis, while $\mathbf{K}_{O, r_{\max }}=692.52$ $\mathrm{Nm} / \mathrm{rad}$, and $\mathbf{K}_{O, r_{\text {min }}}=544.52 \mathrm{Nm} / \mathrm{rad}$ for the $y$ and $z$ axes. These difference between the axes comes from the terms related to the lever arms of the contact points $\left(\mathbf{K}^{\ddagger}\right)$. Indeed, $x$-axis is the one joining both end-effectors of the robots, and therefore, the $\mathbf{K}^{\ddagger}$ term is symmetric between both and cancels itself. Instead, for the $y$ and $z$-axes, both robots' endeffectors are located in the same direction, meaning both exert a force around the same lever arm, being the values increased. Therefore, setting again $30 \%$ of the object's stiffness values rendered $\mathbf{K}_{O, t}=670 \mathrm{~N} / \mathrm{m}$ in all axis, and $\mathbf{K}_{O, r}=46.4$ $\mathrm{Nm} / \mathrm{rad}$ for the $x$-axis, and $\mathbf{K}_{O, r}=588.92 \mathrm{Nm} / \mathrm{rad}$ for the $y$ and $z$-axes.

Fig. 9 shows the graphical results of this third experiment. The user stars in FM control mode, driving the fix-base manipulator end-effector towards the box handle (Fig. 6 pictures 1-2) and grasping it (Fig. 9 at $t \approx 45 \mathrm{~s}$ ). Then, the control mode is changed to MM, and the MOCA platform is displaced towards the box (Fig. 6, picture 3), grasping it from the other handle (Fig. 9 at $t \approx 100 \mathrm{~s}$ ). At that point, the $\mathrm{CM}$ is activated, and both robots synchronously raise the box (Fig. 6, picture 4 ), making the forces in the $z$-axis to raise due to the box weight. The box is then translated in the $y$-axis to place it in the trolley (Fig. 6, pictures 5-7). Once the object is released (Fig. 9 at $t \approx 160 \mathrm{~s}$ ), the user switched back to the FM control mode and moved the Franka robot's end-effector away from the box (Fig. 6, picture 8). We can observe how releasing the box implied an overall reduction of the forces. Finally, the operator took control of the MOCA robot switching to the MM control mode and grasped the trolley's handle to drive it backwards towards the new location (Fig. 6, pictures 9-10). It must be noted how, along the experiment, even if $\mathbf{K}_{O, r}$ was significantly smaller for the $x$-axis, the resulting interaction torques are of the same order of magnitude than for the other axes.

\section{Discussion}

The performed experiments show the capacity of the developed framework to operate with different multi-robot configurations, being the robots working individually or in a cooperative/collaborative manner. These experiments also proved the easiness-of-use of the proposed user interface based on visual feedback and a SpaceMouse. Indeed, though users did not have a complete perception of the 3D environment (2D vision feedback was provided), thanks to the adaptability of the controller and the information provided to the user in


Figure 9: Third experimental results. Interaction forces/torques and Cartesian stiffness values for the collaborative set-up. Resulting end-effector displacement and interaction forces/torques for the fixed-base robot (Franka) and the mobile manipulator (MOCA). Left column: translation; right column: rotation. During this experiment a fix stiffness was selected at the object-level which was distributed to both manipulators according to the control mode (10). Blue, red-, and green-shadowed areas represent the operator using the FM, MM, and CM control modes, respectively.

terms of contact forces and interactions, the subjects were able to successfully perform the tasks. The framework also demonstrated its capability to easily manage different hardware platforms and end-effector devices by means of the virtual hand concept. Hence, the proposed scalable multi-robot tele-impedance control structure can be applied to different scenarios (industrial, medical, and research purposes), being able to adapt to changes and upgrades of the used hardware.

More specifically, from the first set of experiments, we can conclude that being able to regulate the stiffness allowed safer interactions with the patients, as less stiff contacts resulted in lower impact forces. Peak values usually occurred in the approximation phase towards the manikin, before the user stabilized the position and the simulated auscultation period. Few exceptions were observed, with one subject forgetting to modify the stiffness and just one having higher impact forces for lower stiffness. This was due to the operator commanding larger vertical displacements which resulted in the end-effector pushing against the manikin. The subjective tests further supported these data, where most of the subjects reported perceiving the patient to be safer when using self-selected stiffness (with lower values in the direction of interaction).

Nonetheless, the selection of stiffness values was reported to be cumbersome and not intuitive, especially for "naive" users. This translated into a significant increase in the task's execution time (Table I). Several solutions can be thought to improve this issue, among the suggested ones, an interesting approach is to provide the users with a set of few carefully chosen 
combinations of impedance parameters for the different tasks. In this way, the operator would only need to press one button to set the 6 diagonal values of ${ }^{W} \mathbf{K}_{O}$ (6). Still, better results should be expected as users become more familiar with the UI. Similarly, for the next experiments, a webcam was fixed on the robot, and eventually, a second video feedback was used to stream the current situation of the environment. These were still low-quality cameras, regardless of which, users were able to accomplish all the tasks successfully. Therefore, better performance can also be expected by improving the visual feedback.

The second and third experiments served to evidence how the framework can be efficiently used for cooperative and collaborative tasks, allowing to smoothly switch between all control modes, and being able to use the force feedback to detect key instants, e.g., contact with a handle to initiate a grasp operation.

In particular, the second experiment showed how the framework is able to switch between control modes, moving both robots independently while changing their stiffness values. It showed the successful accomplishment of the simulated auscultation task in the cooperation control mode of the framework. We could also observe how increasing the desired stiffness resulted in an increase in the interaction forces/torques, which can be undesirable or even dangerous for the robot in case of surface irregularities. However, these are needed when a precise trajectory tracking is required (e.g., the positioning of the wheelchair). On the contrary, lower stiffness resulted in very low interaction forces/torques.

For the third experiment, we focused on the switching between modes and especially on the capability of the framework to distribute the desired movement and stiffness of the grasped object between the different collaborative robots. A noticeable point is how the limits of the rotational stiffness on the $x$-axis are significantly lower than those on the $y$ and $z$-axes. This results from the symmetry of the contact points, compensating the translation terms in the $x$-axis. Contrarily, around the $y$ and $z$-axis, the translational forces create torques that contribute to the resulting applied torques. Indeed, the minimum stiffness on the $y$ and $z$-axes is $544.54 \mathrm{Nm} / \mathrm{rad}$, which is already a high value, and results from the used $30 \%$ of translational stiffness (together with the minimum established rotational stiffness). While the same equations are used in the $x$-axis, the applied forces around this axis cancel themselves, and therefore only the influence of the minimum set of rotation stiffness applies $(2 \mathrm{Nm} / \mathrm{rad})$. Nonetheless, from the results it can be observed that the interaction torques around $x$-axis are of the same magnitude of those around the $y$ and $z$-axes, meaning that, actually, due to the influence of the translational forces (stiffness), different rotational stiffness values are required to produce similar torques.

\section{CONCLUSiON}

This manuscript presented a scalable framework for teleimpedance control of heterogeneous robots, individually, cooperatively, or in collaboration. The developed method allows the scalability of the solution, being able to apply the same framework for one robot individually, up to any number of robots in collaboration. In this framework, the user focuses on the high-level requirements of the task, defining the desired motions and impedance parameters at the object level. The controller is then responsible for distributing these parameters into the individual robots.

Future work will focus on the implementation of upgrades in terms of the user interface to render the system easier to use and more intuitive. We will also investigate the possibility to add more autonomy to the system, especially for the more repetitive sub-tasks. Finally, we will explore the suggestions made in terms of automatic stiffness regulation, trying to retrieve a set of basic impedance configurations that can be set depending on the task requirements.

\section{APPENDIX}

\section{A. Notation}

Main mathematical symbols and notations used throughout the text are defined in Table III.

Table III: Main mathematical notation

\begin{tabular}{|c|c|}
\hline Symbol & Description \\
\hline $\begin{array}{l}\mathbf{v} \\
\mathbf{v}_{i} \\
\mathbf{v}_{i k}\end{array}$ & $\begin{array}{l}\text { generic vector } \mathbf{v} \in \mathbb{R}^{n} \\
\text { the } i \text {-th element of vector } \mathbf{v} \\
\text { the } i \text {-th element of vector } \mathbf{v} \text { at time instant } k\end{array}$ \\
\hline $\begin{array}{l}\mathbf{V} \\
\mathbf{V}_{i, j} \\
\mathbf{V}_{i, j_{k}}\end{array}$ & $\begin{array}{l}\text { generic matrix } \mathbf{V} \in \mathbb{R}^{m \times n} \\
\text { the element of } \mathbf{V} \text { located at the } i \text {-th row and } j \text {-th colomn } \\
\text { the element of } \mathbf{V} \text { located at the } i \text {-th row and } j \text {-th colomn at } \\
\text { time instant } k\end{array}$ \\
\hline $\begin{array}{l}(\cdot)^{T} \\
(\cdot)_{\min } \\
(\cdot)_{\max } \\
\overline{(\cdot)}\end{array}$ & $\begin{array}{l}\text { transpose operator } \\
\text { the minimum value of the operand }(\cdot) \\
\text { the maximum value of the operand }(\cdot) \\
\text { the average value of the operand }(\cdot)\end{array}$ \\
\hline$\{\cdot\}$ & Cartesian frame- $x y z$ \\
\hline$\{\cdot\}_{k}$ & Cartesian frame- $x y z$ at time instant $k$ \\
\hline$\{O\}$ & object frame \\
\hline$\left\{R_{i}\right\}$ & $\begin{array}{l}\text { base frame of the } i \text {-th robot with respect to which the com- } \\
\text { mands of the end-effector are sent }\end{array}$ \\
\hline$\left\{M_{i}\right\}$ & $\begin{array}{l}\text { the robotic arm's reference frame of the } i \text {-th mobile-base } \\
\text { manipulator }\end{array}$ \\
\hline$\left\{P_{i}\right\}$ & the current location of the $i$-th mobile-base manipulator \\
\hline$\left\{C_{i}\right\}$ & contact point of the $i$-th virtual finger (or robotic module) \\
\hline$\left\{B_{i}\right\}$ & $\begin{array}{l}\text { base of each virtual finger, from which the DH parameters are } \\
\text { defined }\end{array}$ \\
\hline$\{W\}$ & the world (reference) frame of the virtual hand system \\
\hline${ }^{B} \mathbf{T}_{A}$ & transformation matrix from $\{A\}$ to $\{B\}$ \\
\hline${ }^{B} \mathbf{R}_{A}$ & rotation matrix from $\{A\}$ to $\{B\}$ \\
\hline${ }^{B} \boldsymbol{X}_{A}$ & pose of $\{A\}$ expressed in $\{B\}$ \\
\hline $\mathbf{p}(\Delta \mathbf{p})$ & translation (relative) vector along $x y z$ axes \\
\hline$\epsilon(\Delta \epsilon)$ & rotation (relative) vector around $x y z$ axes \\
\hline${ }^{B} \mathbf{K}_{A}$ & Cartesian stiffness matrix in $\{A\}$ expressed in $\{B\}$ \\
\hline
\end{tabular}




\section{B. Virtual hand definition}

The virtual hand used in the experiments is defined with the DH parameters given in Table IV.

Table IV: Virtual hand: fingers' DH parameters. $\theta_{i}, \mathbf{x}_{r}$, and $y_{r}$ represent the different controllable joints of each robot. Note that $d_{f}$ changes depending on the end-effector used in the fixed-base manipulator (mock-up auscultation device or Franka's default gripper)

\begin{tabular}{|cccc|ccccc|}
\hline \multicolumn{3}{|c|}{ Fixed Manipulation (FM) } & \multicolumn{4}{c|}{ Mobile Manipulation (MM) } \\
$\alpha$ & $a$ & $\theta$ & $d$ & $\alpha$ & $a$ & $\theta$ & $d$ \\
\hline$\pi / 2$ & 0.0 & $\theta_{1}$ & 0.333 & $\pi / 2$ & 0.0 & $\pi / 2+\theta_{r}$ & 0.333 \\
$\pi / 2$ & 0.0 & $\theta_{2}$ & 0.0 & $-\pi / 2$ & 0.0 & $-\pi / 2$ & $x_{r}$ \\
$\pi / 2$ & 0.0825 & $\theta_{3}$ & 0.316 & $\pi / 2$ & 0.0 & $-\pi / 2$ & $y_{r}$ \\
$-\pi / 2$ & -0.0825 & $\theta_{4}$ & 0.0 & $-\pi / 2$ & 0.0 & $\theta_{1}$ & 0.333 \\
$\pi / 2$ & 0.0 & $\theta_{5}$ & 0.384 & $\pi / 2$ & 0.0 & $\theta_{2}$ & 0.0 \\
$\pi / 2$ & 0.088 & $\theta_{6}$ & 0.0 & $\pi / 2$ & 0.0825 & $\theta_{3}$ & 0.316 \\
0.0 & -0.13 & $\theta_{7}+\pi / 2$ & $d_{f}{ }^{*}$ & $-\pi / 2$ & -0.0825 & $\theta_{4}$ & 0.0 \\
& & & & $\pi / 2$ & 0.0 & $\theta_{5}$ & 0.384 \\
& & & & $\pi / 2$ & 0.088 & $\theta_{6}$ & 0.0 \\
& & & 0.0 & -0.13 & $\theta_{7}+\pi / 2$ & 0.2020 \\
\hline
\end{tabular}

\section{Cartesian impedance controller}

For the fixed manipulators, we use the Cartesian impedance control of redundant and flexible-joint robots based on [39] (the implementation of this controller can be found in the Franka Emika repository in GitHub page ${ }^{2}$ ):

$$
\begin{aligned}
& \boldsymbol{\tau}_{q}=\boldsymbol{\tau}_{\text {task }}+\boldsymbol{\tau}_{n}+\boldsymbol{\tau}_{c} \\
& \boldsymbol{\tau}_{\text {task }}=\mathbf{J}_{i}^{T}\left(\mathbf{K}_{C_{i}}{ }^{W} \mathbf{X}_{C_{e}}-\mathbf{D}_{C_{i}} \mathbf{J}_{i} \dot{\mathbf{q}}\right) \\
& \boldsymbol{\tau}_{n}=\left(\mathbf{I}_{7 \times 7}-\mathbf{J}_{i}^{T} \mathbf{J}_{i}^{T^{-1}}\right)\left(K_{n}\left(\mathbf{q}_{n}-\mathbf{q}\right)-2 \sqrt{K_{n}} \dot{\mathbf{q}}\right) .
\end{aligned}
$$

$\mathbf{X}_{C, e_{i}}$ and $\mathbf{J}_{i}$ are the Cartesian position error and Jacobian matrix of the $i$-th robot, respectively. $\mathbf{D}_{C_{i}}$ represents the damping parameters. As we consider the stiffness as a diagonal matrix (Section II-B, these are given by $\mathbf{D}_{C_{i}}=0.25 \sqrt{\mathbf{K}_{C_{i}}}$. If non-diagonal matrices are used for the stiffness, other methods such as double-diagonalization can be used to retrieve the damping parameters. $\tau_{c}$ and $\tau_{n}$ are the Coriolis and nullspace terms. While the first is given by the robot internal controller, the second is computed, where $\mathbf{K}_{n}$ is the nullspace stiffness constant. In our experiments we set $\mathbf{K}_{n}=15 \mathrm{Nm} / \mathrm{rad}$.

For the case of mobile manipulators, the whole-body impedance controller in [27] is employed. A similar equation to the first one in (15) is used, where the virtual torques corresponding to the MOCA robot must be translated into desired velocity by means of:

$$
\mathbf{M}_{\mathrm{adm}} \ddot{\mathbf{q}}_{v}^{\mathrm{des}}+\mathbf{D}_{\mathrm{adm}} \dot{\mathbf{q}}_{v}^{\mathrm{des}}=\boldsymbol{\tau}_{v}^{\mathrm{vir}}+\boldsymbol{\tau}_{v}^{\mathrm{ext}} .
$$

$\mathbf{M}_{\text {adm }} \in \mathbb{R}^{m \times m}$ and $\mathbf{D}_{\text {adm }} \in \mathbb{R}^{m \times m}$ are the virtual inertia and damping of the robot, $\dot{\mathbf{q}}_{v}^{\mathrm{des}} \in \mathbb{R}^{m}$ is the desired velocity to be sent to the platform, and $\boldsymbol{\tau}_{v}^{\mathrm{vir}} \in \mathbb{R}^{m}$ and $\boldsymbol{\tau}_{v}^{\text {ext }} \in \mathbb{R}^{m}$ are the computed virtual and external torque interfaces, respectively.

\section{REFERENCES}

[1] S. Avgousti, E. G. Christoforou, A. S. Panayides, S. Voskarides, C. Novales, L. Nouaille, C. S. Pattichis, and P. Vieyres, "Medical telerobotic systems: current status and future trends," Biomedical engineering online, vol. 15, no. 1, p. 96, 2016.

[2] M. Loffler, N. Costescu, E. Zergeroglu, and D. Dawson, "Telerobotic decontamination and decommissioning with qrobot, a pc-based robot control system," International Journal of Computers and Applications, vol. 24, no. 3, pp. 112-121, 2002.

${ }^{2}$ https://github.com/frankaemika/franka_ros/
[3] M. Barsotti, F. Stroppa, N. Mastronicola, S. Marcheschi, and A. Frisoli, "Teleoperated bilateral-arm rehabilitation with alex rehab station," in International Conference on NeuroRehabilitation, pp. 185-189, Springer, 2018.

[4] N. G. Tsagarakis, D. G. Caldwell, F. Negrello, W. Choi, L. Baccelliere, V.-G. Loc, J. Noorden, L. Muratore, A. Margan, A. Cardellino, et al., "Walk-man: A high-performance humanoid platform for realistic environments," Journal of Field Robotics, vol. 34, no. 7, pp. 1225-1259, 2017.

[5] B. Weber, R. Balachandran, C. Riecke, F. Stulp, and M. Stelzer, "Teleoperating robots from the international space station: Microgravity effects on performance with force feedback," in IEEE International Conference on Intelligent Robots and Systems, pp. 8138-8144, IEEE, 2019.

[6] X. Xu, B. Cizmeci, C. Schuwerk, and E. Steinbach, "Model-mediated teleoperation: toward stable and transparent teleoperation systems," IEEE Access, vol. 4, pp. 425-449, 2016.

[7] A. Ajoudani, N. Tsagarakis, and A. Bicchi, "Tele-impedance: Teleoperation with impedance regulation using a body-machine interface," The International Journal of Robotics Research, vol. 31, no. 13, pp. 1642 1656, 2012.

[8] M. Laghi, A. Ajoudani, M. G. Catalano, and A. Bicchi, "Unifying bilateral teleoperation and tele-impedance for enhanced user experience," The International Journal of Robotics Research, vol. 39, no. 4, pp. 514539, 2020.

[9] W. Fu, M. M. van Paassen, D. A. Abbink, and M. Mulder, "Framework for human haptic perception with delayed force feedback," IEEE Transactions on Human-Machine Systems, vol. 49, no. 2, pp. 171-182, 2018.

[10] C. Neupert, S. Matich, N. Scherping, M. Kupnik, R. Werthschützky, and C. Hatzfeld, "Pseudo-haptic feedback in teleoperation," IEEE transactions on haptics, vol. 9, no. 3, pp. 397-408, 2016.

[11] M. Laghi, M. Maimeri, M. Marchand, C. Leparoux, M. Catalano, A. Ajoudani, and A. Bicchi, "Shared-autonomy control for intuitive bimanual tele-manipulation," in 2018 IEEE-RAS 18th International Conference on Humanoid Robots (Humanoids), pp. 1-9, IEEE, 2018.

[12] M. Koga, K. Kosuge, K. Furuta, and K. Nosaki, "Coordinated motion control of robot arms based on the virtual internal model," IEEE Transactions on Robotics and Automation, vol. 8, pp. 77-85, Feb 1992.

[13] T. Wimbock, C. Ott, and G. Hirzinger, "Impedance behaviors for twohanded manipulation: Design and experiments," in Proceedings 2007 IEEE International Conference on Robotics and Automation, pp. 41824189, April 2007.

[14] S. A. Schneider and R. H. Cannon, "Object impedance control for cooperative manipulation: theory and experimental results," IEEE Transactions on Robotics and Automation, vol. 8, no. 3, pp. 383-394, 1992.

[15] Y. Nakamura, K. Nagai, and T. Yoshikawa, "Mechanics of coordinative manipulation by multiple robotic mechanisms," in Proceedings. 1987 IEEE International Conference on Robotics and Automation, vol. 4, pp. 991-998, 1987.

[16] R. C. Bonitz and T. C. Hsia, "Internal force-based impedance control for cooperating manipulators," IEEE Transactions on Robotics and Automation, vol. 12, no. 1, pp. 78-89, 1996.

[17] T. Wojtara, M. Uchihara, H. Murayama, S. Shimoda, S. Sakai, H. Fujimoto, and H. Kimura, "Human-robot collaboration in precise positioning of a three-dimensional object," Automatica, vol. 45, no. 2, pp. 333 - 342, 2009.

[18] R. Ikeura, T. Moriguchi, and K. Mizutani, "Optimal variable impedance control for a robot and its application to lifting an object with a human," in Proceedings. 11th IEEE International Workshop on Robot and Human Interactive Communication, pp. 500-505, Sep. 2002.

[19] A. Mörtl, M. Lawitzky, A. Kucukyilmaz, M. Sezgin, C. Basdogan, and S. Hirche, "The role of roles: Physical cooperation between humans and robots," The International Journal of Robotics Research, vol. 31, no. 13, pp. 1656-1674, 2012

[20] M. Laghi, M. Maimeri, M. Marchand, C. Leparoux, M. Catalano, A. Ajoudani, and A. Bicchi, "Shared-autonomy control for intuitive bimanual tele-manipulation," in 2018 IEEE-RAS 18th International Conference on Humanoid Robots (Humanoids), pp. 1-9, 2018.

[21] Z. Gharaybeh, H. Chizeck, and A. Stewart, Telerobotic Control in Virtual Reality. IEEE, 2019.

[22] J. I. Lipton, A. J. Fay, and D. Rus, "Baxter's homunculus: Virtual reality spaces for teleoperation in manufacturing," IEEE Robotics and Automation Letters, vol. 3, no. 1, pp. 179-186, 2017.

[23] F. Brizzi, L. Peppoloni, A. Graziano, E. Di Stefano, C. A. Avizzano, and E. Ruffaldi, "Effects of augmented reality on the performance of teleoperated industrial assembly tasks in a robotic embodiment," IEEE 
Transactions on Human-Machine Systems, vol. 48, no. 2, pp. 197-206, 2017.

[24] A. Yew, S. Ong, and A. Nee, "Immersive augmented reality environment for the teleoperation of maintenance robots," Procedia Cirp, vol. 61, pp. 305-310, 2017.

[25] D. Sun, A. Kiselev, Q. Liao, T. Stoyanov, and A. Loutfi, "A new mixedreality-based teleoperation system for telepresence and maneuverability enhancement," IEEE Transactions on Human-Machine Systems, vol. 50, no. 1, pp. 55-67, 2020.

[26] D. Dajles, F. Siles, et al., "Teleoperation of a humanoid robot using an optical motion capture system," in 2018 IEEE International Work Conference on Bioinspired Intelligence (IWOBI), pp. 1-8, IEEE, 2018.

[27] Y. Wu, P. Balatti, M. Lorenzini, F. Zhao, W. Kim, and A. Ajoudani, "A teleoperation interface for loco-manipulation control of mobile collaborative robotic assistant," IEEE Robotics and Automation Letters, vol. 4, no. 4, pp. 3593-3600, 2019.

[28] K. Darvish, Y. Tirupachuri, G. Romualdi, L. Rapetti, D. Ferigo, F. J. A. Chavez, and D. Pucci, "Whole-body geometric retargeting for humanoid robots," in 2019 IEEE-RAS 19th International Conference on Humanoid Robots (Humanoids), pp. 679-686, IEEE, 2019.

[29] E.-J. Rolley-Parnell, D. Kanoulas, A. Laurenzi, B. Delhaisse, L. Rozo, D. G. Caldwell, and N. G. Tsagarakis, "Bi-manual articulated robot teleoperation using an external rgb-d range sensor," in 2018 15th International Conference on Control, Automation, Robotics and Vision (ICARCV), pp. 298-304, IEEE, 2018.

[30] M. Martins, A. Cunha, and L. Morgado, "Usability test of 3dconnexion $3 \mathrm{~d}$ mice versus keyboard+ mouse in second life undertaken by people with motor disabilities due to medullary lesions," Procedia Computer Science, vol. 14, pp. 119-127, 2012.

[31] D. A. Lawrence, "Stability and transparency in bilateral teleoperation," IEEE transactions on robotics and automation, vol. 9, no. 5, pp. 624637, 1993.

[32] M. R. Cutkosky and I. Kao, "Computing and controlling compliance of a robotic hand," IEEE Transactions on Robotics and Automation, vol. 5, pp. 151-165, April 1989.

[33] D. Prattichizzo and J. C. Trinkle, Grasping in the Springer Handbook of Robotics. Springer, July 2016.

[34] A. Bicchi, "On the problem of decomposing grasp and manipulation forces in multiple whole-limb manipulation," Robotics and Autonomous Systems, vol. 13, pp. 127 - 147, Jul 1994.

[35] "SpaceMouse Compact." https://www.3dconnexion.com/spacemouse_ compact/en/. Accessed: 2020-07-06.

[36] N. Corporation, "Qt, a cross platform application and UI framework," 2012.

[37] T. Wimbock, C. Ott, and G. Hirzinger, "Impedance behaviors for twohanded manipulation: Design and experiments," in Proceedings 2007 IEEE International Conference on Robotics and Automation, pp. 41824189, April 2007.

[38] A. Albu-Schäffer, C. Ott, and G. Hirzinger, "A unified passivity-based control framework for position, torque and impedance control of flexible joint robots," The international journal of robotics research, vol. 26, no. 1 , pp. 23-39, 2007.

[39] C. Ott, Cartesian impedance control of redundant and flexible-joint robots. Springer, 2008.

[40] A. Dietrich, K. Bussmann, F. Petit, P. Kotyczka, C. Ott, B. Lohmann, and A. Albu-Schäffer, "Whole-body impedance control of wheeled mobile manipulators," Autonomous Robots, vol. 40, no. 3, pp. 505-517, 2016.

[41] S. Haddadin and S. Parusel, "Franka emika panda," 2018.

[42] M. G. Catalano, G. Grioli, E. Farnioli, A. Serio, M. Bonilla, M. Garabini, C. Piazza, M. Gabiccini, and A. Bicchi, "From soft to adaptive synergies: The Pisa/IIT softhand," in Human and Robot Hands, pp. 101-125, Springer, 2016.

[43] NASA, "NASA TLX test." https://humansystems.arc.nasa.gov/groups/ TLX/, 2011. [Online; accessed 25-February-2020].

[44] M. Malvezzi, G. Gioioso, G. Salvietti, D. Prattichizzo, and A. Bicchi, "Syngrasp: A matlab toolbox for grasp analysis of human and robotic hands," in 2013 IEEE International Conference on Robotics and Automation, pp. 1088-1093, IEEE, 2013.

[45] V. Ruiz Garate, "Syngrasp multirobot." https://github.com/Virginia5632/ syngrasp, 2020.

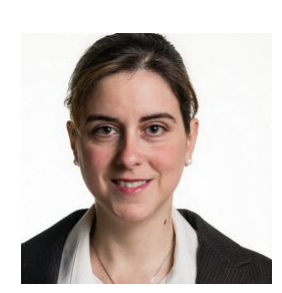

Virginia Ruiz Garate is a PostDoc at the HumanRobot Interfaces and Physical Interaction $\left(\mathrm{HRI}^{2}\right)$ laboratory of the Istituto Italiano di Tecnologia (IIT) in Italy. She obtained her PhD in 2016 from the Universite Catholique de Louvain (UCL) in Belgium. She has been serving as a reviewer for IEEE journals and conferences such as RA-L, ICRA, IROS, BioRob, etc. She co-organized the RSS 2019 workshop on "Emerging Paradigms for Robotic Manipulation: from the Lab to the Productive World" from which a RAM SI developed. In the $\mathrm{HRI}^{2}$ group she worked for the SOMA project studying new grasping paradigms, and she is now researching under the SOPHIA project. Her current research interests include grasping and manipulation, bio-inspired control, assistive robotics, and human-robot collaboration.

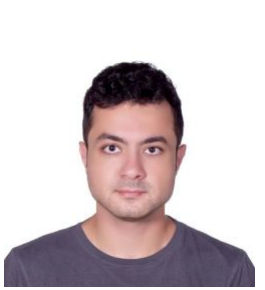

Soheil Gholami obtained both his Bachelor of Science (2013) and Master of Science (2016) degrees in Electrical Engineering with a focus on Control Engineering. Currently, he is pursuing a Ph.D. in Bioengineering at Politecnico di Milano in the Neuroengineering and Medical Robotics Laboratory (NearLab), working in collaboration with Istituto Italiano di Tecnologia (IIT) at the Human-Robot Interfaces and physical Interaction (HRI) laboratory, Genova, Italy. His research is mainly focused on the development and implementation of advanced techniques and interfaces for dynamic human-robot interaction and collaboration.

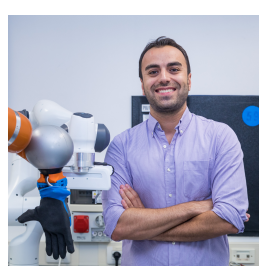

Arash Ajoudani is a tenured senior scientist at the Italian Institute of Technology (IIT), where he leads the Human-Robot Interfaces and physical Interaction $\left(\mathrm{HRI}^{2}\right)$ laboratory. He received his $\mathrm{PhD}$ degree in Robotics and Automation from University of Pisa and IIT in July 2014. His PhD thesis was a finalist for the Georges Giralt $\mathrm{PhD}$ award 2015 - best European $\mathrm{PhD}$ thesis in robotics. He is a recipient of the European Research Council (ERC) starting grant 2019. He was a winner of the Amazon Research Awards 2019, the winner of the Solution Award 2019 (Premio Innovazione Robotica at MECSPE2019), the winner of the KUKA Innovation Award 2018, the winner of the Werob best poster award 2018, a finalist for the best conference paper award at Humanoids 2018, a finalist for the best interactive paper award at Humanoids 2016, a finalist for the best oral presentation award at Automatica (SIDRA) 2014, the winner of the best student paper award and a finalist for the best conference paper award at ROBIO 2013, and a finalist for the best manipulation paper award at ICRA 2012. He is the coordinator of the Horizon-2020 project SOPHIA with a consortium of 12 partners from the leading European research and industrial organisations. He has contributed to several successful European projects (H2020 and FP7) such as WALKMAN, WearHap, SOMA, and SoftPro. $\mathrm{He}$ is the author of the book "Transferring Human Impedance Regulation Skills to Robots" in the Springer Tracts in Advanced Robotics (STAR), and several publications in journals, international conferences, and book chapters. $\mathrm{He}$ is currently serving as the executive manager of the IEEE-RAS Young Reviewers' Program (YRP), and as chair and representative of the IEEE-RAS Young Professionals Committee. He has been serving as a member of scientific advisory committee and as an associate editor for several international journals and conferences such as IEEE RAL, Biorob, ICORR, etc. His main research interests are in physical human-robot interaction and cooperation, robotic manipulation, robust and adaptive control, assistive robotics, and tele-robotics. 